Please send trade news information and

illustrations to Arveen Bajaj at the BDJ, Nature

Publishing Group, The Macmillan Building,

4-6 Crinan Street, London N1 9XW.

Trade news is provided as a service to readers

using text and images from the manufacturer,

supplier or distributor and does not imply

endorsement by the BDJ. Normal and prudent

research should be exercised before purchase or

use of any product mentioned.

\section{Lasting restorations}

Practice Plan, in association with Sirona Dental Systems Ltd and Medenta, is offering all of their practices an opportunity to purchase the chairside CAD/ CAM restorative device at an attractive price.

Available to Practice Plan customers, direct from Sirona, the Cerec 3 Complete means you can now provide your patients with strong, lasting restorations without the need for temporaries, in just one appointment. The system means fewer injections, less drilling and less time in the chair for your patients and it can reduce your overhead costs, reduce cancelled appointments and increase profits.

The added value package for Practice Plan customers includes preferential finance, with 12 months interest free credit then a special low rate, a materials voucher worth $£ 1,000$ and free insurgery and external training.

Reader response number 51

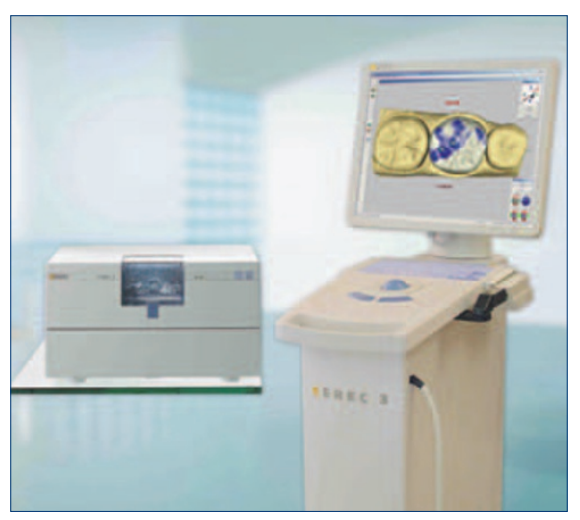

\title{
Reduce tripping hazards
}

Dentsply has recently added two new ultrasonic scaling units to its Cavitron range.

The wireless foot pedal means having one less wire in the surgery, helps towards reducing tripping hazards and you can place the control exactly where you need to. The foot control can also be pressed from any side and does not flip over. There are two depression settings on the pedal allowing a boost of power to overcome any tenacious build-up.

Another new feature, which helps you to better manage infection control and saves you time, is the purge setting. All you have to do is remove the insert, place the handpiece over a sink and press the purge button to automatically flush out the system for two minutes before you use it on the next patient.

\section{Easy ordering}

Henry Schein presents the new Orthodontic Product Guide offering ortho essentials from treatment supplies to braces for GDPs and orthodontists alike, all under the Henry Schein Brand. The range of products on offer includes vital orthodontic treatment supplies, appliances and braces and which come with a full 12-month money back guarantee.

To help make your ordering quicker and easier, every product has been barcoded ready for you to use with your eZ Scan scanner. Simply scan the barcode of the product you wish to order via your

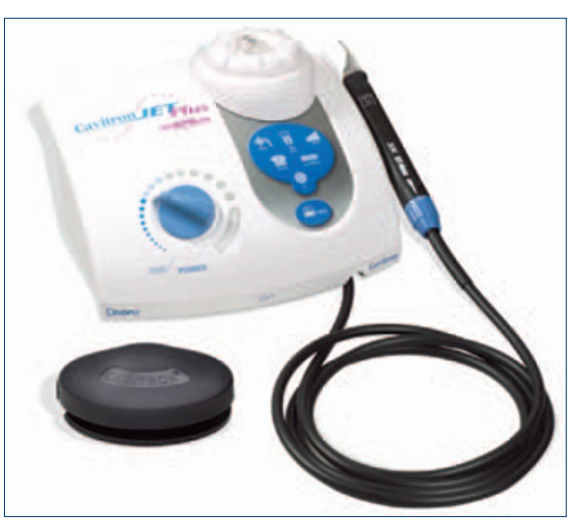

These new features are available on both the new units: Cavitron Plus, which is the ultrasonic scaler and the Cavitron Jet Plus, which is the combined 2-in-1 air polisher and ultrasonic scaler. Reader response number 50

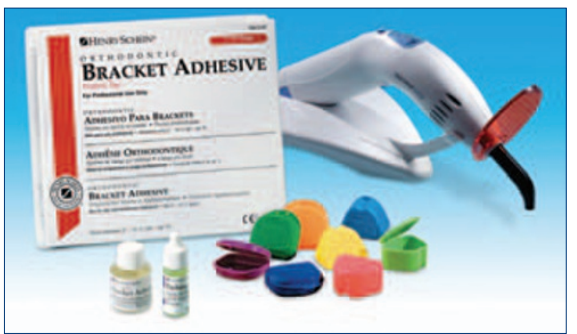

Henry Schein eZ scan scanner and then scan the quantity required.

Then by using your existing electronic ordering method at www.henryschein. co.uk or Aruba eZ, connect the scanner to your PC to upload your order.

Reader response number 52

\section{Cement that won't wash out}

Myerson's NexTemp is designed to combat problems that can occur when using eugenol based temporary cements, which can interfere with the setting of composite materials.

Zinc oxide has a tendency to wash out when left in situ for a period in time, which can in turn lead to secondary decay. Premier NexTemp is a resin based cement and does not contain either eugenol or zinc oxide, making it suitable for all temporary situations be they long or short term.

The chlorhexidine component of NexTemp acts as an antibacterial agent and the potassium nitrate part is a known desensitiser that sedates the pulp.

Reader response number 53

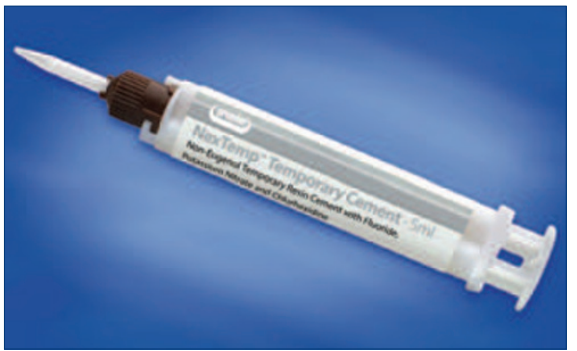




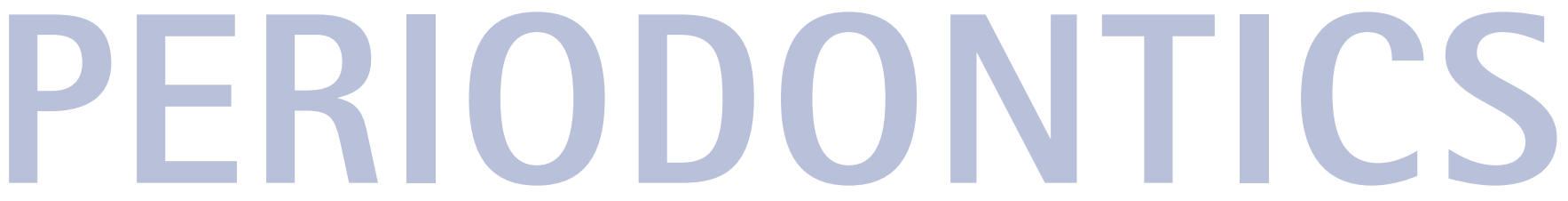

\section{Fluoride release}

GC's new Fuji IX Extra is a packable, aesthetic glass ionomer restorative. Releasing six times the amount of fluoride compared to a conventional glass ionomer, it is a truly translucent material.

Until now this type of glass ionomer has been confined to the posterior region due to difficulties in achieving a natural, life like appearance.

Its transluceny combined with its shade matching qualities allows Fuji IX Extra to be used in the front of the mouth where aesthetics are of course important.

Being a glass ionomer the product adheres to the tooth structure without the need for etching and bonding materials and indeed the material performs better in a slightly damp working environment.

Its chemical setting enables it to be placed in a single layer, no matter the thickness. Indicated for more difficult situations such as paediatric dentistry, the elderly, those patients with restricted mouth opening and patients prone to caries, Fuji IX Extra is easy to place with minimal cavity preparations required.

Reader response number 55

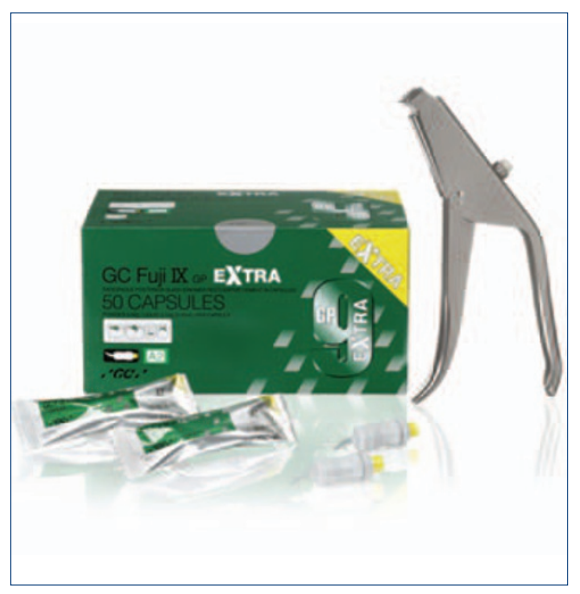

\section{Plaque fighting}

Among Curaprox's range of products is the SR (Straight Ready) brush range. The SR brush selection complements the existing range, is obtainable in five colour-coded widths to accommodate every patient and designed to effectively penetrate at-risk interdental spaces and help to keep them free of plaque.

Reader response number 54

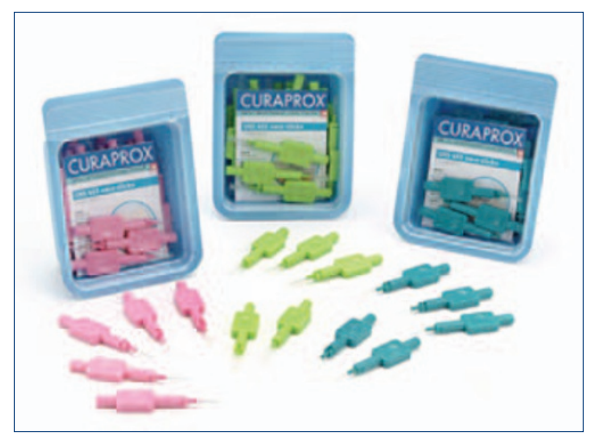

\section{Protecting your patients}

GC's Saliva Testing kit is designed to test the saliva at both resting and stimulated states, as the characteristics of these forms of saliva are different. The kit can be an effective tool in assisting with early detection of the condition of the oral environment, according to the company.

It adds that diagnostics can be greatly enhanced in terms of early detection of oral problems, with improved patient communication and motivation com-

\section{Predictable re-growth}

Straumann's Emdogain is a treatment solution to periodontal disease. A biology-based product, it promotes the predictable re-growth of hard and soft tissues lost due to periodontal disease. Emdogain saves treatment time for both practitioner and patient, allowing rapid placement and little time in manipulation of the material into a periodontal defect, resulting in a limited exposure of open tissues. It does not affect tissue integrity beyond that of normal flap surgery and requires only one surgical procedure. No follow-up surgery is necessary.

Practitioners can benefit from Emdogain's convenience and effectiveness when used in areas difficult to treat such

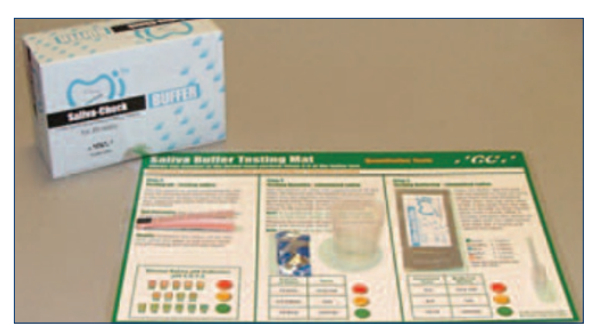

bined with an increased dental awareness of your patients.

Reader response number 56

as interproximal areas, defects distal to the second molar, defects located under bridgework and wide defects.

Reader response number 57

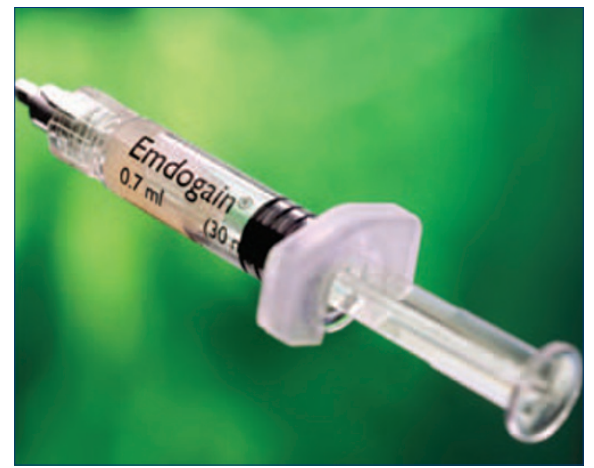




\section{Colour-style prophylaxes set}

Trycare Dental Linkline are the UK Distributors for Zepf Instruments, which includes their new Colour-Style Prophylaxes Set. The set comprises all the most popular prophylaxis instruments, ie a complete set of Gracey Curettes (5-6, 7-8, 11-12 and 13-14), a Sickle Scaler H6/7, a Gracey Sickle Scaler 204S, a Probe Head

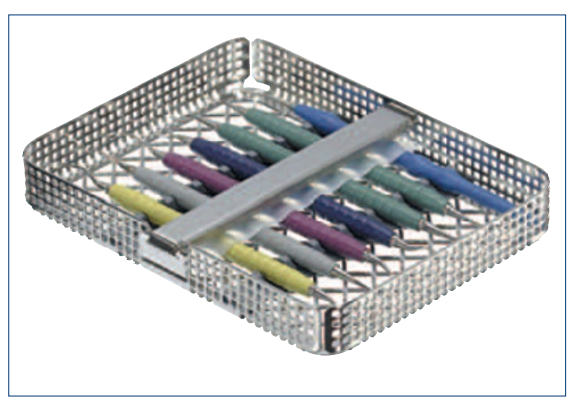

M2.5 and a Universal handle for probes, mirrors and explorers.

Extremely light, which enhances the operators 'feel', and ergonomically designed, Colour-Style Instruments are manufactured with a special Zepf Bright Surface Finish, which makes instrument identification, particularly between the Gracey Currettes, much simpler and quicker.

Suitable for ultrasonic cleaning and autoclaving, the Complete Set is supplied in $1 / 2$ sized stainless steel wash tray, which holds eight instruments, and incorporates two silicone rubbers and an instrument retainer to hold them secure during the cleaning cycle and transportation. Every item is available separately too.

Reader response number 58

\section{Effective bone regeneration}

Fortoss is a range of bone regeneration products available exclusively from The Dental Directory. There are four products in total - Resorb, Cema, Perma and Vital.

Fortoss Resorb is a versatile material that can be used around immediate implants, sinuses, dehiscences and extraction sockets where implants are indicated.

Fortoss Cema is an easy to use resorbable membrane that is applied as a paste and sets under the soft tissues. It is compatible with any augmentation material. Fortoss Perma is ideal for maintaining ridge height and width after extraction or to build bone lost to peri-implantitis. Lastly, there's Fortoss Vital, a synthetic bone regenerative that mimics the min-

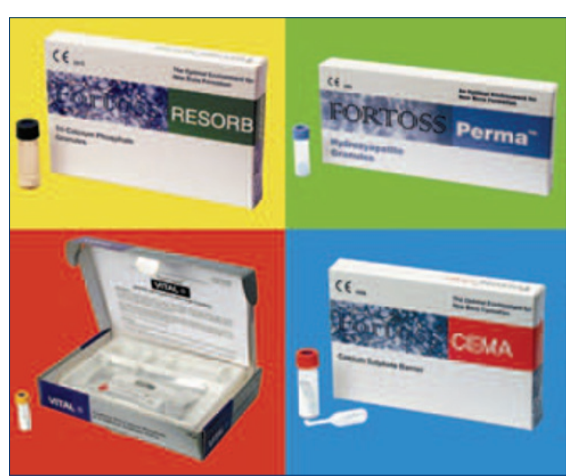

eral phase of human bone. It is ideal for periodontal use or surgical endodontics as well as around implants, sinuses, dehiscences and extraction pockets.

Reader response number 60

\section{Simplified shade matching}

The Clearfil family of adhesive and restorative products includes Clearfil Esthetic Cement with simplified shade matching and ease-of-use. For ambitious ceramic and veneer restorations, Kuraray Dental now presents a new resin cement in several shades. Clearfil Esthetic Cement combines the strength and long term experience of the classic cement Panavia, with the aesthetic spirit of the new dental age.

With its unique shade matching system, the cement simplifies colour selection, allowing you to easily choose a shade by developing four essential colour families, which are arranged by restorative type.

Designed with ease of use in mind, a compact Automix syringe system and five realistic shades with identical try-in pastes offer adhesion with a high bond strength and a whole new level of colour to every type of restoration.

Superior mechanical properties, a high filler load, and a tough self-etching seal make Clearfil Esthetic Cement a highly effective and workable material.

Reader response number $\mathbf{6 2}$

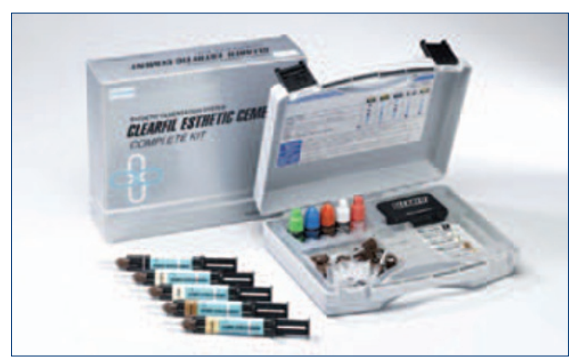

\section{Improved grip}

Flexichange from Dentsply has a handle that is designed to fit comfortably into your hand, with a wide grip near the working ends, and a narrow centre. The wider working grip, softer handle, and dimpled design contribute to reduce hand fatigue, stop the need to 'pinch' the instrument while working, and improve grip and rotational control.

Reader response number 59

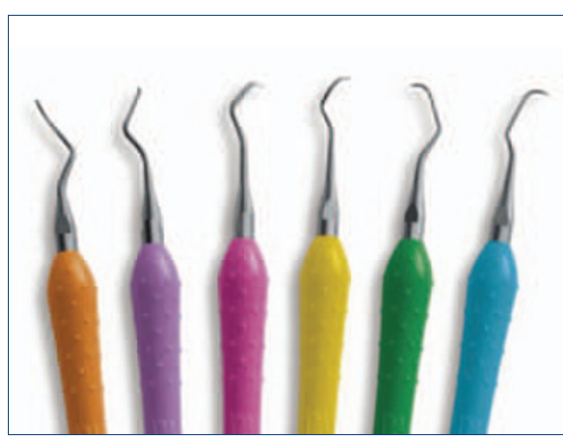

Treatment for chronic periodontitis

Periostat, distributed by Alliance Pharmaceuticals, was recently introduced to the dental market for the treatment of chronic periodontitis. It is an orally administered tablet containing a novel formulation of $23.08 \mathrm{mg}$ doxycycline hyclate. Designed to be taken twice a day as an adjunct to scaling and root planning (SRP), Periostat increases clinical attachment level gain and reduces probing depths in patients with chronic periodontitis.

The duration of treatment with Periostat, a prescription only medicine, will depend on the individual case but is recommended to be at least three months, and its use is approved for up to nine months to suppress destructive collagenase enzymes and help stabilise the periodontium. Periostat is not intended to eliminate the need for clinical interventions or procedures that reduce the bacterial load in the periodontal pocket but is an adjunctive therapy.

Reader response number 61

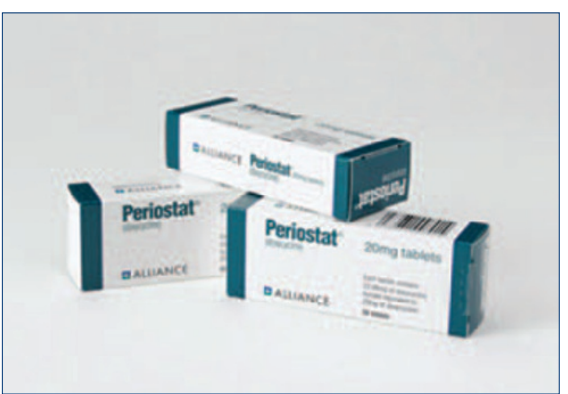

